\title{
Solid Acid-Catalyzed Cellulose Hydrolysis Monitored by In Situ ATR-IR Spectroscopy
}

\author{
J. Zakzeski (Utrecht University) \\ R.J.H. Grisel (ECN) \\ A.T. Smit (ECN) \\ B.M. Weckhuysen (Utrecht \\ University)
}

November 2012

ECN-W--12-041 


\title{
Solid Acid-Catalyzed Cellulose Hydrolysis Monitored by In Situ ATR-IR Spectroscopy
}

\author{
Joseph Zakzeski, ${ }^{[a]}$ Ruud J. H. Grisel, ${ }^{*[b]}$ Arjan T. Smit, ${ }^{[b]}$ and Bert M. Weckhuysen ${ }^{*[a]}$
}

The solid acid-catalyzed hydrolysis of cellulose was studied under elevated temperatures and autogenous pressures using in situ ATR-IR spectroscopy. Standards of cellulose and pure reaction products, which include glucose, fructose, hydroxymethylfurfural (HMF), levulinic acid (LA), formic acid, and other compounds, were measured in water under ambient and elevated temperatures. A combination of spectroscopic and HPLC analysis revealed that the cellulose hydrolysis proceeds first through the disruption of the glycosidic linkages of cellulose to form smaller cellulose molecules, which are readily observed by their distinctive $\mathrm{C}-\mathrm{O}$ vibrational stretches. The continued disruption of the linkages in these oligomers eventually results in the formation and accumulation of monomeric glucose. The solid-acid catalyst accelerated the isomerization of glucose to fructose, which then rapidly reacted under hydrothermal conditions to form degradation products, which included HMF, LA, formic acid, and acetic acid. The formation of these species could be suppressed by decreasing the residence time of glucose in the reactor, reaction temperature, and contact with the metal reactor. The hydrolysis of regenerated cellulose proceeded faster and under milder conditions than microcrystalline cellulose, which resulted in increased glucose yield and selectivity.

\section{Introduction}

Biomass is an important feedstock for the production of fuels, chemicals, and energy. In the United States and the European Union, the highest proportion of renewable energy is obtained from biomass, which surpasses hydropower, geothermal, wind, and solar power. Moreover, biomass is currently the only renewable source of liquid transportation fuels. ${ }^{[1,2]}$ Based on the continued depletion of fossil fuel reserves and the continued increase in chemical and energy demand, the use of biomass can only be expected to increase in the foreseeable future. One particular opportunity to satisfy the energy and raw material requirements of society is through the valorization of lignocellulosic biomass, which is inexpensive, abundant, and renewable. Comprising the inedible component of most plant matter, lignocellulosic biomass has the added advantage that it does not directly compete with agricultural food production. The annual production of lignocellulosic biomass is enormous and, with only modest changes in land use, capable of satisfying virtually all of the raw material requirements of the chemical industry provided that suitable processes for its selective conversion can be developed. ${ }^{[3,4]}$

This impetus has prompted immense interest in the development of processes that readily convert the components of lignocellulosic biomass, namely, cellulose, hemicellulose, and lignin, into useful fuels and chemicals. In particular cellulose, the most abundant component, ${ }^{[5]}$ has attracted considerable attention. Cellulose is a recalcitrant material and has poor solubility in most common solvents, which can be largely explained by its crystalline structure that is energetically stabilized by a vast network of inter- and intramolecular hydrogen bonding. This extensive bonding also protects cellulose from chemical transformation by hindering the access of catalysts, enzymes, and other reagents to key structural components.
Disrupting the innate crystalline structure in a controlled manner is, therefore, critical for the valorization of cellulose.

One method to disrupt the crystalline structure in cellulose is through the use of ionic liquids. Pioneering work by Rogers et al. revealed that certain ionic liquids, such as 1-butyl-3-methylimidazolium chloride, were capable of dissolving cellulose. ${ }^{[6]}$ Chloride anions penetrated the cellulose structure and disrupted the network of hydrogen bonds, and several catalytic processes have since been developed for cellulose valorization using this ionic liquid as the solvent. ${ }^{[7-10]}$ Dissolution of cellulose in an ionic liquid and subsequent precipitation using an antisolvent, such as water or methanol, results in significant alterations to the cellulose microenvironment. The material obtained as a result of this pretreatment process, termed regenerated cellulose, is significantly more susceptible to conversion by enzymatic or catalytic processes, such as hydrolysis, relative to untreated cellulose. ${ }^{[11,12]}$

Of the catalytic processes, hydrolysis is particularly attractive as a method to produce glucose, which is a useful platform chemical from which many other valuable chemicals can be

\footnotetext{
[a] Dr. J. Zakzeski, Prof. Dr. B. M. Weckhuysen

Inorganic Chemistry and Catalysis Group, Utrecht University

Universiteitsweg 99, 3584 CG Utrecht, (The Netherlands)

Fax: $(+31) 302-534328$

E-mail:b.m.weckhuysen@uu.nl

[b] Dr. R. J. H. Grisel, A. T. Smit

Biomass, Coal \& Environmental Research

Energy Research Centre of the Netherlands (ECN)

Westerduinweg 3, PO Box 1, 1755 ZG Petten, (The Netherlands)

Fax: $(+31) 224-568487$

E-mail:grisel@ecn.nl

Supporting Information for this article is available on the WWW under http://dx.doi.org/10.1002/cssc.201100631.
} 
made. The hydrolysis of cellulose to glucose and subsequent conversion to products such as hydroxymethylfurfural (HMF) and levulinic acid (LA) have been reported using surface-supported Lewis-acid, Brønsted-acid ${ }^{[6]}$ and solid-acid catalysts ${ }^{[13-20]}$ in water. Kinetic and mechanistic studies on the conversion of cellulose derivates and sugars to HMF and LA have provided considerable insight into the types of transformations that occur in these systems. ${ }^{[21,22]}$ Often, the performance of these processes depends strongly on reaction conditions, and strategic selection of the appropriate conditions vastly influences product yield and selectivity. The ability to monitor the changes that occur during a process under the operating conditions is especially advantageous to maximize product conversion and selectivity.

The products that are formed during the conversion of cellulose to glucose and sequential degradation, such as HMF or LA, contain a wide range of acid, aldehyde, and alcohol functional groups, and the characteristic vibrations associated with these functional groups are detectable by using IR spectroscopy. The principle challenges in the online monitoring of these processes by using IR spectroscopy arise not only from the elevated temperatures and pressures under which these processes occur, but also because of the strong absorptions of the solvent, particularly bulk water, which often interfere with detection of the relevant products and reactants.

Attenuated total reflection (ATR)-IR spectroscopy has been described as a promising technique for monitoring chemical reactions on line. ${ }^{[23-25]}$ Because most of the absorption comes from the first few layers that are in contact with the probe ${ }^{[26]}$ rather than from the bulk, ATR-IR spectroscopy circumvents the interference caused by the strong water absorptions, ${ }^{[18]}$ which allows the observation of the vibrations associated with the substrate and product functional groups. To the best of our knowledge, only ATR-IR spectroscopic studies of cellulose involved the determination of cellulose structural characteristics or cellulose-water interactions. ${ }^{[27,28]}$ In this work, we demonstrate the use of an autoclave reactor equipped with an ATR-IR spectroscopic probe as a useful means to monitor the transformations that occur during the solid-acid-catalyzed hydrolysis of cellulose in water in situ.

\section{Results and Discussion}

A possible route through which glucose and the various other chemicals are formed by the acid-catalyzed hydrolysis of cellulose is depicted in Scheme 1. The steps in this route are supported by previous mechanistic studies on acid-catalyzed cellulose hydrolysis ${ }^{[21,22]}$ and the spectroscopic results presented below. The vibrations and the assignments associated with these species are presented in Table $1 .{ }^{[29]}$ The process begins with the cellulose starting material, which is hydrolyzed in the presence of the solid-acid catalyst and water to form cellulose with a reduced chain length. The increased solubility of this material relative to the starting cellulose allows the observation of several vibrations, which include the most prominent $\mathrm{C}-\mathrm{O}$ stretch and $\mathrm{C} 1-\mathrm{H}$ bend at $\tilde{v}=1105$ and $1026 \mathrm{~cm}^{-1}$, respectively. Continued hydrolysis results in the formation and accumulation of glucose, which is observed by vibrations at $\tilde{v}=1074$ and $1038 \mathrm{~cm}^{-1}$. Isomerization of glucose to fructose and subsequent dehydration results in the formation of HMF, which is best observed by the $\mathrm{C}=\mathrm{C}$ stretch at $\tilde{v}=1586 \mathrm{~cm}^{-1}$. The HMF aldehyde vibration is not as pronounced under the reaction conditions likely because of the reaction of water with the aldehyde to form a geminal diol, the equilibrium of which is thermodynamically favored at elevated temperatures. The appearance of a vibration at around $\tilde{v}=1710 \mathrm{~cm}^{-1}$ indicates the formation of LA and formic acid (or other acid species), which form as a result of HMF hydrolysis.

\section{Pure component analysis}

The IR spectra of several pure standards, which are the expected products of cellulose hydrolysis, such as glucose, fructose, HMF, LA, acetic acid, and formic acid, were measured in water at room and reaction temperature (typically $423 \mathrm{~K}$ ) using the ATR-IR spectroscopic probe. The results for glucose, the targeted product for cellulose hydrolysis, are given in Figure 1. The most intense vibrations both at room and reaction temperature appeared at approximately $\tilde{v}=1034$ and $1079 \mathrm{~cm}^{-1}$, which were assigned to $\mathrm{C} 1-\mathrm{H}$ and $\mathrm{C}-\mathrm{O}$ bends, respectively. ${ }^{[2]}$ Other prominent vibrations included $\mathrm{C}-\mathrm{O}$ stretching vibrations

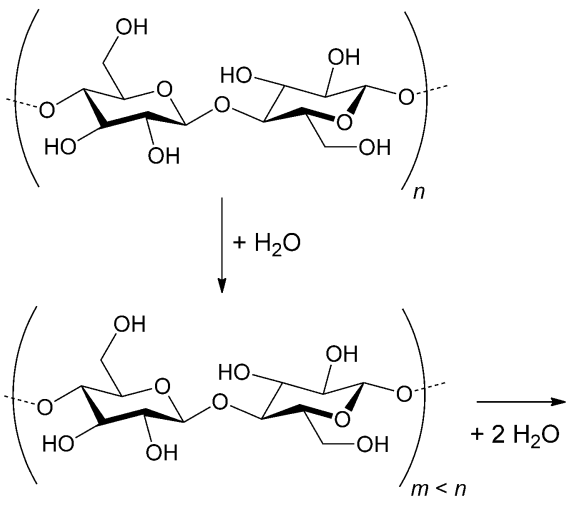

A

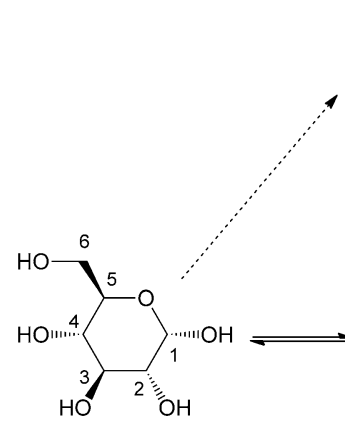

B

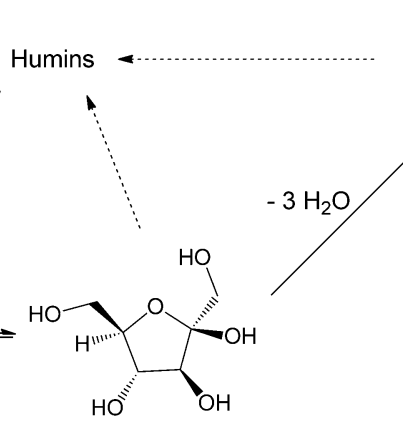

C

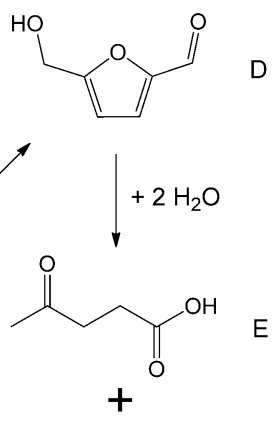

$\mathrm{OH}_{\mathrm{OH}}^{\mathrm{O}}$

Scheme 1. Proposed reaction pathway for cellulose hydrolysis. 
Table 1. Selected vibrations and assignments of species depicted in Scheme 1.

\begin{tabular}{|c|c|c|c|c|c|c|c|}
\hline \multicolumn{2}{|c|}{ Species } & \multirow{2}{*}{$\begin{array}{l}\begin{array}{l}\text { Frequency } \\
{\left[\mathrm{cm}^{-1}\right]}\end{array} \\
\approx 1213\end{array}$} & \multirow{2}{*}{$\begin{array}{l}\text { Assignment } \\
\text { multiple vibrations }\end{array}$} & \multicolumn{2}{|c|}{ Species } & \multirow{2}{*}{$\begin{array}{l}\begin{array}{l}\text { Frequency } \\
{\left[\mathrm{cm}^{-1}\right]}\end{array} \\
1664\end{array}$} & \multirow{2}{*}{$\begin{array}{l}\text { Assignment } \\
\mathrm{C}=\mathrm{O} \text { ald }^{[\mathrm{a}]} \mathrm{str}\end{array}$} \\
\hline$A$ & cellulose & & & D & HMF & & \\
\hline & & 1161 & $\mathrm{C}-\mathrm{O} \mathrm{str}^{[\mathrm{b}]}$ & & & 1586 & $\mathrm{C}=\mathrm{C}$ str \\
\hline & & 1105 & $\mathrm{C}-\mathrm{O}$ str & & & 1556 & $\mathrm{C}=\mathrm{C}$ str \\
\hline & & 1026 & $\mathrm{C} 1-\mathrm{H}$ bend & & & 1526 & $\mathrm{C}=\mathrm{C}$ str \\
\hline \multirow[t]{7}{*}{ B } & glucose & 1396 & $\mathrm{C} 2-\mathrm{H}$ bend & & & 1400 & $\mathrm{C}=\mathrm{C}$ str \\
\hline & & 1362 & $\mathrm{C}-\mathrm{H}$ bend & & & 1194 & ring $=\mathrm{C}-\mathrm{O}-\mathrm{C}=$ def \\
\hline & & 1153 & $\mathrm{C}-\mathrm{O}$ str & & & 1060 & $\mathrm{C}-\mathrm{H} \mathrm{def}^{[\mathrm{c}]}$ \\
\hline & & 1101 & $\mathrm{C}-\mathrm{O}$ str & & & 1022 & ring def \\
\hline & & 1074 & $\mathrm{C}-\mathrm{O}, \mathrm{C}-1-\mathrm{H}$ bend & & & 970 & $\mathrm{C}-\mathrm{H}$ op ${ }^{[\mathrm{d}]} \mathrm{def}$ \\
\hline & & 1038 & $\mathrm{C} 1-\mathrm{H}$ bend & & & 799 & $\mathrm{C}-\mathrm{H}$ op def \\
\hline & & 900 & $\mathrm{C}-\mathrm{O}-\mathrm{C}$ bend & $E$ & $\mathrm{LA}$ & 1710 & $\mathrm{C}=\mathrm{O}$ acid str \\
\hline \multirow[t]{6}{*}{ C } & fructose & 1415 & $\mathrm{C}-\mathrm{H}$ bend & & & $1631^{[\mathrm{e}]}$ & $\mathrm{C}=\mathrm{O}$ ald str \\
\hline & & 1347 & $\mathrm{C}-\mathrm{H}$ bend & & & 1407 & $\mathrm{CH}_{3}$ asym bend \\
\hline & & 1258 & multiple vibrations & & & 1369 & $\mathrm{CH}_{3}$ sym bend \\
\hline & & 1157 & $\mathrm{C}-\mathrm{O}$ str & & & 1172 & $\mathrm{C}-\mathrm{O}$ acid str \\
\hline & & 1086 & $\mathrm{C}-\mathrm{O}$ str & $\mathrm{F}$ & formic acid & 1710 & $\mathrm{C}=\mathrm{O}$ acid str \\
\hline & & 1064 & $\mathrm{C}-\mathrm{H}$ bend & & & 1195 & $\mathrm{C}-\mathrm{O}$ acid str \\
\hline
\end{tabular}

$1407 \mathrm{~cm}^{-1}$, respectively, and the symmetric bending vibrations for both compounds appear at $\tilde{v}=1370 \mathrm{~cm}^{-1}$. These results indicate that the observation of the formation of these acids is possible by tracking the appearance of the acid vibration at $\tilde{v}=$ $1710 \mathrm{~cm}^{-1}$, but identification of the acid species requires observation of the vibrations that appear between $\tilde{v}=1410$ and $1170 \mathrm{~cm}^{-1}$.

The IR spectra of HMF at room temperature and $443 \mathrm{~K}$ are given in Figure S4 in the Supporting Information. At room temperature, a vibration assigned to a $\mathrm{C}=\mathrm{O}$ aldehyde stretch was observed at $\tilde{v}=1664 \mathrm{~cm}^{-1}$. A series of vibrations in the range ex-

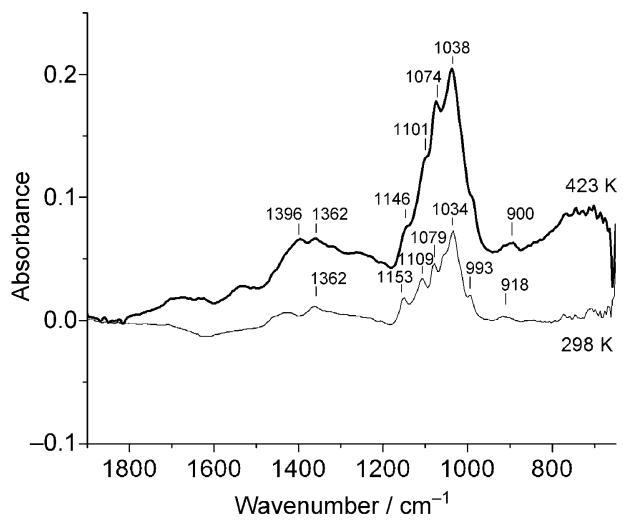

Figure 1. IR spectra of glucose measured in water. Spectra at $423 \mathrm{~K}$ offset for clarity.

at $\tilde{v}=1153$ and $1109 \mathrm{~cm}^{-1}$, weaker vibrations assigned to a $\mathrm{C}-\mathrm{O}-\mathrm{C}$ bend at $\tilde{v}=918 \mathrm{~cm}^{-1}$, and $\mathrm{C} 2-\mathrm{H}$ vibrations at $\tilde{v}=1396$ and $1362 \mathrm{~cm}^{-1}$. Heating the solution to $423 \mathrm{~K}$ resulted in an increase in the vibrational intensity of several bands, especially at $\tilde{v}=1038$ and $1074 \mathrm{~cm}^{-1}$; however, the frequency of these vibrations remained almost unchanged at elevated temperatures. In the region between $\tilde{v}=4000$ and $1900 \mathrm{~cm}^{-1}$ (not shown), weak vibrations assigned to $\mathrm{C}-\mathrm{H}$ stretches $(\tilde{v}=$ $\approx 2930 \mathrm{~cm}^{-1}$ ) and strong, broad water $\mathrm{O}-\mathrm{H}$ stretching vibrations $\left(\tilde{v}=3700-3000 \mathrm{~cm}^{-1}\right)$ were also observed.

The Supporting Information contains the IR spectra acquired for LA, acetic acid, and formic acid, which all exhibited a $\mathrm{C}=\mathrm{O}$ acid stretch at around $\tilde{\nu}=1710 \mathrm{~cm}^{-1}$. The vibration from the ketone component of LA appeared at $\tilde{v}=1631 \mathrm{~cm}^{-1}$ at room temperature, but was not observed at elevated temperatures, possibly as a result of the formation of a geminal diol. The $\mathrm{C}-$ $\mathrm{O}$ acid stretch for formic acid, acetic acid, and LA appeared at $\tilde{v}=1213,1280$, and $1172 \mathrm{~cm}^{-1}$, respectively. The acetic acid and $\mathrm{LA} \mathrm{CH}_{3}$ asymmetric bends are observed at $\tilde{v}=1396$ and pected for $C=C$ stretches were observed at $\tilde{v}=1586,1526$, and $1400 \mathrm{~cm}^{-1}$. A $=\mathrm{C}-\mathrm{O}-\mathrm{C}=$ ring vibration was detected at $\tilde{v}=$ $1194 \mathrm{~cm}^{-1}$, a $\mathrm{C}-\mathrm{H}$ in-plane deformation at $\tilde{v}=1071 \mathrm{~cm}^{-1}$, a ring deformation at $\tilde{v}=970 \mathrm{~cm}^{-1}$, and $\mathrm{C}-\mathrm{H}$ out-of-plane deformations at $\tilde{v}=970$ and $772 \mathrm{~cm}^{-1}$. Upon heating to $443 \mathrm{~K}$, the $\mathrm{C}=\mathrm{C}$ vibrations at $\tilde{v}=1586$ and $1556 \mathrm{~cm}^{-1}$ became significantly more pronounced, and the appearance of vibrations that resemble those of $\mathrm{LA}$, which include $\mathrm{C}=\mathrm{O}$ and $\mathrm{C}-\mathrm{O}$ acid stretches at $\tilde{v}=1706$ and $1161 \mathrm{~cm}^{-1}$, respectively, suggested that HMF decomposes to LA under hydrothermal conditions. ${ }^{[21,22]}$

The vibrational frequencies of fructose, which is likely formed as an intermediate during HMF formation, exhibited several similar vibrations as observed with glucose, particularly at $\tilde{v}=1415,1347$, and $1157 \mathrm{~cm}^{-1}$. The most intense fructose vibrations appeared at $\tilde{v}=1064$ and $1086 \mathrm{~cm}^{-1}$, which are shifted to slightly higher wavenumbers than those observed with glucose. Fructose was considerably less stable than glucose at elevated temperatures even in the absence of a catalyst. The time-resolved IR spectra of the reaction of fructose in water at $423 \mathrm{~K}$ are given in Figure 2, which shows the changes in vibrational intensity with time. The spectrum recorded of the fructose solution in water at room temperature was used as a reference and was subtracted from the other spectra to emphasize the changes that occur during heating. The most significant vibrations appeared at $\tilde{v}=1707$ and $1586 \mathrm{~cm}^{-1}$, which corresponded to the formation of acids and HMF (see above). These vibrations increased in intensity with time and those associated with fructose (around $\tilde{v}=1056 \mathrm{~cm}^{-1}$ ) decreased in intensity. HPLC analysis of the solution after $3 \mathrm{~h}$ (Table 2 , entry 1 ) revealed the presence of acetic acid (3.2\%), formic acid (2.3\%), LA (1.2\%), and relatively large amounts of HMF (11.2\%), which supported the spectroscopic results. Large quantities of unknown products were detected, which may include unconverted fructose and soluble condensation products formed between HMF and fructose. A small amount of solid material was 


\begin{tabular}{|c|c|c|c|c|c|c|c|c|c|c|}
\hline Entry & Substrate & $\begin{array}{l}\text { Glucose } \\
{[\%]}\end{array}$ & $\begin{array}{l}\text { 2-Furalde- } \\
\text { hyde [\%] }\end{array}$ & $\begin{array}{l}\mathrm{HMF} \\
{[\%]}\end{array}$ & $\begin{array}{l}\text { Acetic } \\
\text { acid [\%] }\end{array}$ & $\begin{array}{l}\text { Formic } \\
\text { acid [\%] }\end{array}$ & $\begin{array}{l}\text { LA } \\
{[\%]}\end{array}$ & $\begin{array}{l}\text { Unknown } \\
\text { [\%] }\end{array}$ & $\begin{array}{l}\text { Recovered solid } \\
\text { material }^{[a]}[\%]\end{array}$ & $\begin{array}{l}\text { Glucose } \\
\text { selectivity [\%] }\end{array}$ \\
\hline $1^{[c, d]}$ & D-fructose & 0.0 & 0.3 & 11.2 & 3.2 & 2.3 & 1.2 & 78.9 & 2.9 & 0.0 \\
\hline $2^{[e, f, g]}$ & $\alpha$-D-glucose & 18.8 & 0.2 & 1.8 & 0.5 & 3.2 & 12.4 & 62.4 & 0.0 & 18.6 \\
\hline $3^{[\mathrm{h}]}$ & $\alpha$-D-glucose & 78.6 & 0.0 & 0.4 & 0.0 & 0.4 & 0.0 & 20.6 & 0.0 & 78.6 \\
\hline $4^{[c, h]}$ & $\alpha$-D-glucose & 90.6 & 0.0 & 4.7 & 0.0 & 0.0 & 0.0 & 3.5 & 1.2 & 91.7 \\
\hline 6 & cellulose & 46.6 & 0.7 & 3.4 & 0.4 & 0.8 & 1.9 & 19.1 & 27.2 & 63.9 \\
\hline $7^{[]]}$ & cellulose & 17.1 & 0.2 & 0.5 & 0.0 & 0.9 & 0.0 & 27.4 & 53.9 & 37.1 \\
\hline $8^{[j]}$ & cellulose & 54.1 & 0.0 & 1.7 & 0.3 & 0.6 & 0.8 & 27.8 & 14.8 & 63.5 \\
\hline
\end{tabular}

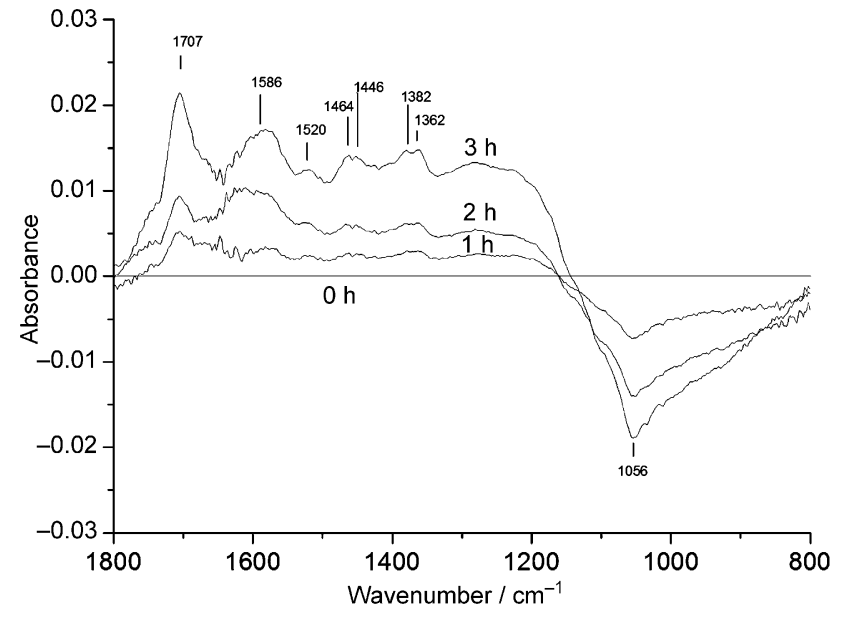

Figure 2. Time-resolved differential IR spectra of the reaction of D-fructose in water. Reaction conditions: D-fructose $(0.31 \mathrm{~g}), \mathrm{H}_{2} \mathrm{O}(25 \mathrm{~g}), 423 \mathrm{~K}$.

collected, but no glucose was detected in the reaction mixture at the conclusion of the reaction.

\section{Reactions of glucose with solid-acid catalysts}

The time-resolved IR spectra of glucose in water in the presence of a sulfated zirconia catalyst (SZR) are given in Figure 3.

Upon reaching reaction temperature [time $(t)=0 \mathrm{~h}$ ], the most prominent vibrations were those associated with glucose at $\tilde{v}=1074$ and $1038 \mathrm{~cm}^{-1}$, which decreased in intensity as the reaction proceeded. A weak vibration appeared in the acid region at $\tilde{v}=1710 \mathrm{~cm}^{-1}$, and the intensity of the vibration increased with time. Moreover, vibrations at $\tilde{v}=1407$ and $1366 \mathrm{~cm}^{-1}$ were observed, which could be attributed to either formic acid or the $\mathrm{LA} \mathrm{CH}_{3}$ asymmetric and symmetric bends, respectively. A vibration at $\tilde{v}=1175 \mathrm{~cm}^{-1}$ resembled the $\mathrm{C}-\mathrm{O}$ acid stretch in LA. A weak vibration at $\tilde{v}=1593 \mathrm{~cm}^{-1}$ also increased slightly with increasing reaction time, which was attributed to HMF accumulation (see above). HPLC results at the conclusion of the reaction indicated relatively high LA concentrations (12.4\%) based on initial carbon (Table 2, entry 2). Other products included glucose (18.8\%), formic acid (3.2\%),

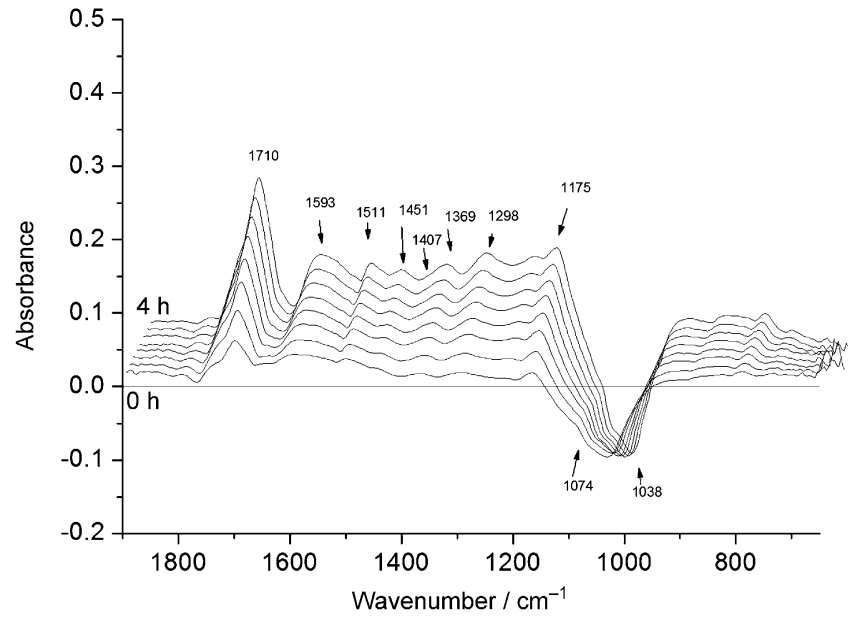

Figure 3. Time-resolved differential IR spectra of the acid-catalyzed hydrolysis of glucose in water. Reaction conditions: D-glucose $(5 \mathrm{~g})$, SZR $(0.58 \mathrm{~g})$, $\mathrm{H}_{2} \mathrm{O}(25 \mathrm{~g}), 453 \mathrm{~K}$.

acetic acid $(0.5 \%)$, and small quantities of HMF (1.8\%), fructose $(0.3 \%)$, mannose $(0.4 \%)$, and 2-furaldehyde $(0.2 \%)$. Glucose likely degraded to (in)soluble humins and other products not detected by HPLC, which accounts for the relatively high amount of unknown products. The analytical results corresponded well with the vibrations observed by using ATR-IR spectroscopy, with the most prominent vibrations corresponding to the formation of LA and consumption of glucose.

The results of glucose degradation in the presence of the activated carbon catalyst at $423 \mathrm{~K}$ are given in Table 2, entry 3. Under these milder conditions, a larger proportion of the original glucose was recovered at the conclusion of the reaction. Small quantities of HMF and formic acid were detected, in addition to a large quantity of unknown products (20.6\%). The analytical results of glucose hydrolysis under similar conditions, but in the absence of a catalyst, are given in Table 2, entry 4. Under these conditions, more than $90 \%$ of the glucose starting material was recovered. HMF (4.7\%) and small quantities of solid material were observed, but in less than half the amount compared to fructose. In the absence of a catalyst, no acid products were detected. Moreover, a significant reduction in 
the quantity of unknown products was observed in the absence of a catalyst. These results suggest that the catalyst accelerates the formation of glucose degradation by-products.

\section{Acid-catalyzed hydrolysis of cellulose}

The hydrothermal treatment of regenerated cellulose was studied at $423 \mathrm{~K}$ in the absence of a catalyst. The time-resolved ATR-IR spectra are given in Figure 4. The spectrum of the cellulose solution in water at room temperature was used as a reference and was subtracted from the other spectra to emphasize the changes that occurred during heating. The only peaks observed in the reference spectrum were attributed to water

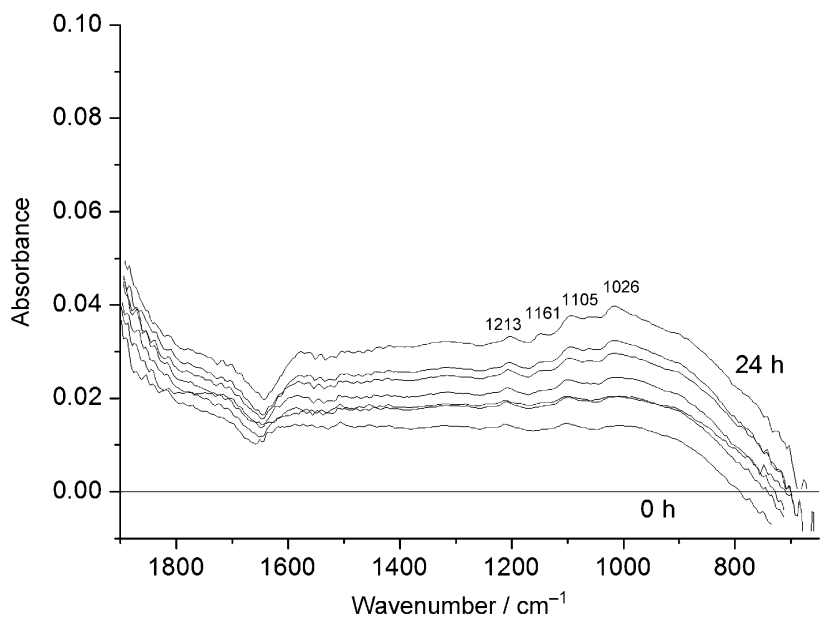

Figure 4. Time-resolved differential IR spectra of cellulose in water. Reaction conditions: regenerated cellulose $(0.25 \mathrm{~g}), \mathrm{H}_{2} \mathrm{O}(25 \mathrm{~g}), 423 \mathrm{~K}$.

vibrations, which included a broad peak at $\tilde{v}=3335 \mathrm{~cm}^{-1}$, assigned to the water $\mathrm{O}-\mathrm{H}$ stretch, and a peak at $\tilde{v}=1639 \mathrm{~cm}^{-1}$, assigned to the water $\mathrm{O}-\mathrm{H}$ bend. Peaks associated with cellulose were not observed because of its insolubility in water. Heating the regenerated cellulose/water mixture to $423 \mathrm{~K}$ resulted in the appearance of very weak vibrations at $\tilde{v}=1213$, 1161,1105 , and $1026 \mathrm{~cm}^{-1}$, which grew slightly in intensity with time. These vibrations differ to those assigned to glucose (see above), and instead are likely to arise from the slow, partial hydrothermal depolymerization of cellulose to smaller cellulose chains that are slightly more water soluble and, therefore, more readily detected by using ATR-IR spectroscopy. In agreement with the spectroscopic results, HPLC analysis of the solution after treatment indicated the presence of only trace quantities of glucose $(0.2 \%$ based on initial carbon), and similarly small quantities of other products, that is, formic acid $(0.3 \%)$, acetic acid $(0.1 \%)$, and HMF $(0.9 \%)$ were detected after $24 \mathrm{~h}$ (Table 2, entry 5). Relatively low solid-substrate mass losses occurred, which corresponded to $19.1 \%$ of the starting material. The discrepancy between the detected materials and residual recovered solid cellulose likely corresponds to the formation of water-soluble, oligomeric glucose that was not detected by using HPLC. Similar results were observed using nonregenerated microcrystalline Avicel cellulose, except that considerably lower conversions were observed $(<5 \%)$, and virtually no change in absorption intensity was observed after the solution had reached reaction temperature, which indicates that very little cellulose hydrolysis occurred in the absence of a catalyst.

The time-resolved ATR-IR spectra of the regenerated cellulose hydrolysis in the presence of a solid-acid catalyst are given in Figure $5 \mathrm{a}$, and the change in absorbance of selected
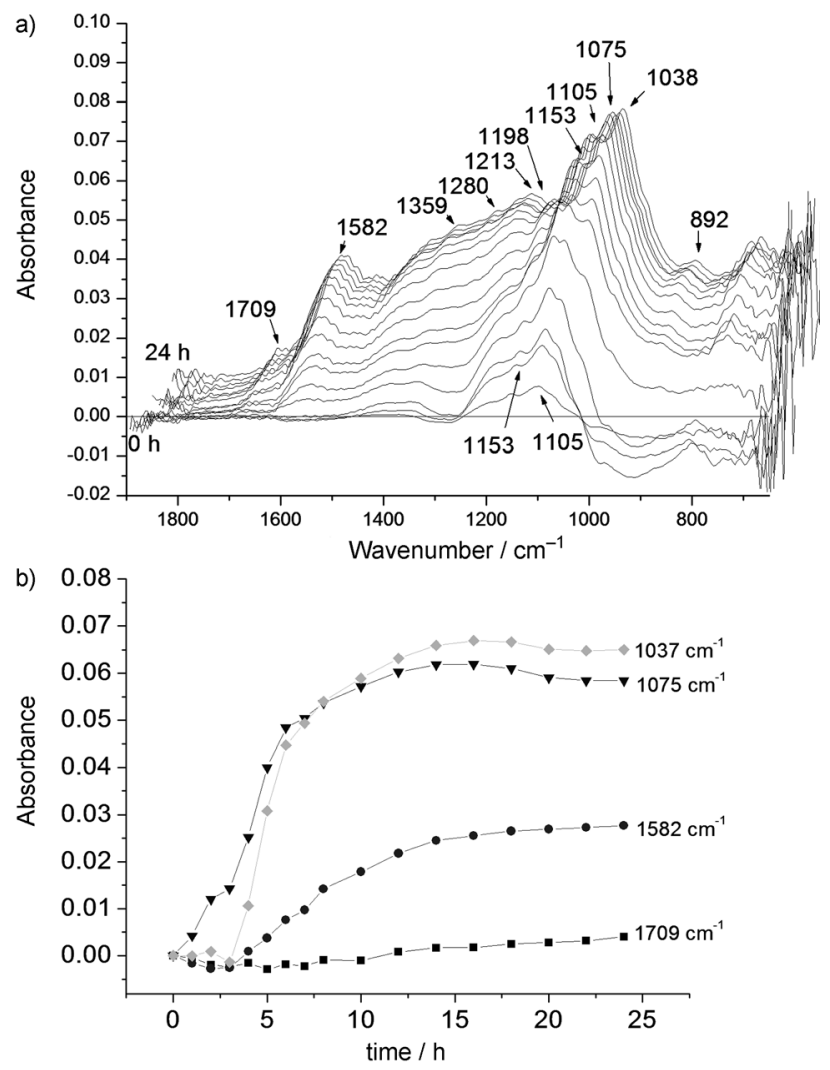

Figure 5. a) Time-resolved differential IR spectra of the solid acid-catalyzed hydrolysis of cellulose in water. Reaction conditions: regenerated cellulose $(0.25 \mathrm{~g})$, active carbon catalyst $\left.(0.25 \mathrm{~g}), \mathrm{H}_{2} \mathrm{O}(25 \mathrm{~g}), 423 \mathrm{~K} . \mathrm{b}\right)$ Change in absorbance of selected vibrations vs. time.

vibrations are given in Figure $5 \mathrm{~b}$. In contrast to the uncatalyzed reaction, significant changes in the IR spectra were observed. In the initial $3 \mathrm{~h}$ at reaction temperature, the most prominent vibrations were observed at $\tilde{v}=1105$ and $1153 \mathrm{~cm}^{-1}$, which continued to grow in intensity with increasing reaction time. These vibrations resemble the weak vibrations observed during heating in the absence of a catalyst except that the intensity increased at a significantly faster rate. After approximately $4 \mathrm{~h}$, additional vibrations that strongly resemble the vibrations observed with pure samples of glucose in water (see above) began to appear at $\tilde{v}=1075,1038$, and $892 \mathrm{~cm}^{-1}$ and continued to increase in intensity until about $16 \mathrm{~h}$, at which point the intensities began to decrease slightly. The appearance of these vibrations also coincided with the appearance of vibrations near $\tilde{v}=1582 \mathrm{~cm}^{-1}$, which also in- 
creased in intensity with time. These vibrations likely correspond to the formation and accumulation of HMF, which exhibits $C=C$ vibrations in this region. After $4 \mathrm{~h}$, vibrations in the aldehyde and acid region at approximately $\tilde{v}=1709 \mathrm{~cm}^{-1}$ began to appear and became significant after 9-10 h. These results are indicative of the formation of LA and possibly formic acid, both of which have a $\mathrm{C}=\mathrm{O}$ acid vibration at around $\tilde{v}=1710 \mathrm{~cm}^{-1}$. HPLC analysis of the solution after the reaction revealed high solid-substrate mass losses corresponding to $72.8 \%$ of the starting material, confirmed the presence of high quantities of glucose $(46.6 \%)$, in agreement with the large changes observed in the IR spectra (Table 2, entry 6). LA $(1.9 \%)$, formic acid $(0.8 \%)$, and acetic acid $(0.4 \%)$ were also detected in the solution along with $\operatorname{HMF}(3.4 \%)$ and 2-furaldehyde $(0.7 \%)$. Small quantities of insoluble humins were also observed. Taken together, the spectroscopic and analytical results suggest that oligomeric glucose (i.e., cellulose with reduced chain length) is formed from cellulose from the time the system reaches reaction temperature. After approximately $4 \mathrm{~h}$, monomeric glucose and some HMF begin to appear in the reactor, and several more hours are required until sufficient acid concentrations are present to allow their detection.

The cellulose starting material, which is insoluble in the aqueous solution, was initially not observed in the ATR-IR spectra most likely because of poor contact of the cellulose with the probe. In the absence of a catalyst, the glycosidic linkages in cellulose remained largely intact; hence, significant changes were not observed in the IR spectra. In the presence of a suitable catalyst, these linkages are susceptible to disruption, and the initial vibrations observed by using ATR-IR spectroscopy are attributed to smaller cellulose fragments that formed through hydrolysis of some of the interior glycosidic linkages in the original cellulose material. Continued hydrolysis of the glycosidic bonds in the smaller fragments eventually resulted in the formation of glucose, the accumulation of which is signified by the vibrations at $\tilde{v}=1075$ and $1038 \mathrm{~cm}^{-1}$. The $\mathrm{C} 1-\mathrm{H}$ vibrations of the larger fragments, which still contain a considerable quantity of glycosidic linkages, are distinct from the vibrations observed with glucose, which lack these linkages and allow the rate of glucose formation to be monitored by using in situ ATR-IR spectroscopy.

The formation of HMF, which proceeds rapidly with the increased accumulation of glucose in the reaction solution, likely proceeds through the formation of fructose from glucose as suggested by previous mechanistic studies on HMF formation. ${ }^{[16,17]}$ Previous studies have indicated that the dehydration of fructose to form HMF occurs rapidly, ${ }^{[16]}$ which supports the very low concentrations of fructose observed at the conclusion of the reaction and the spectroscopic results depicted above. The strongest fructose vibrations, which appear at $\tilde{v}=1086$ and $1064 \mathrm{~cm}^{-1}$, overlap significantly with those from the cellulose fragments and glucose, which precludes the direct observation of these materials by using ATR-IR spectroscopy. The delay in the formation of the acids, indicated by the vibrations at $\tilde{v}=1710 \mathrm{~cm}^{-1}$, supports earlier reports that HMF is an intermediate product in acid-catalyzed glucose degradation. ${ }^{[16,17]}$ These spectroscopic results suggest that, under the given con- ditions, prevention of the degradation of glucose to degradation products, such as HMF, cannot be avoided completely, but the formation of acids and other degradation products can be suppressed by operating at shorter reaction times. Reducing the reaction temperature (Table 2 , entry 7 ) results in slightly reduced byproduct formation, with the glucose/byproduct yield ratio increasing from 6.5 at $423 \mathrm{~K}$ to 10.9 at $413 \mathrm{~K}$ (Figure S7 in the Supporting Information). Both the overall glucose yield and selectivity are lower at 413 than at $423 \mathrm{~K}$, and a higher proportion of unknown products has been detected. These unknown products likely consist of soluble glucose oligomers that were not completely depolymerized under the milder reaction conditions, which is supported by the presence of strong vibrations at $\tilde{v}=1061$ and $1027 \mathrm{~cm}^{-1}$.

\section{Reactions of microcrystalline cellullose}

The solid acid-catalyzed hydrolysis of nonregenerated microcrystalline Avicel cellulose typically requires higher operating temperatures $(>443 \mathrm{~K})$ for the reaction to proceed than required for regenerated cellulose. At elevated temperatures, the presence of stainless steel, from which the ATR-IR sentinel is constructed, may contribute to the decomposition of glu$\operatorname{cose}^{[30]}$ as a decreased glucose stability was observed and relatively large amounts of insoluble humins were formed at $453 \mathrm{~K}$. A teflon liner was used to minimize contact between the solution and the reactor walls. Although the stainless steelpromoted degradation was not as prominent with the regenerated cellulose at lower temperatures, the use of a teflon liner nevertheless resulted in higher overall glucose yields and a glucose/byproduct ratio of 16.4 (Table 2, entry 8). Minimizing contact of the solution with the metal is, therefore, necessary for high glucose selectivity, especially at higher temperatures.

The time-resolved ATR-IR spectra of Avicel cellulose at $453 \mathrm{~K}$ are given in Figure 6. Only very weak vibrations were observed at $\tilde{v}=1071$ and $1026 \mathrm{~cm}^{-1}$ until the solution reached $453 \mathrm{~K}$, at which point a maximum vibrational intensity was observed.

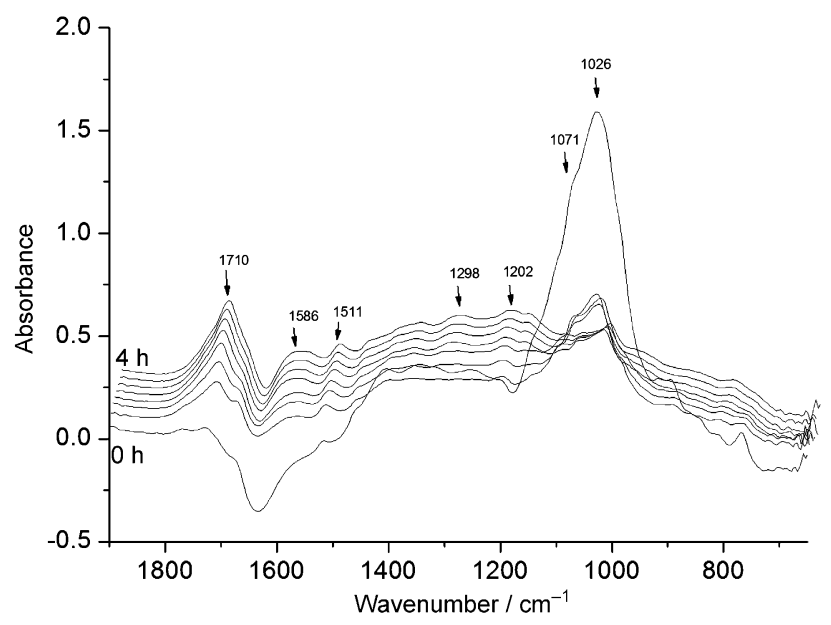

Figure 6. Time-resolved differential IR spectra of the acid-catalyzed hydrolysis of cellulose in water. Reaction conditions: Avicel cellulose $(5 \mathrm{~g}), \mathrm{SZR}$ $(0.58 \mathrm{~g}), \mathrm{H}_{2} \mathrm{O}(25 \mathrm{~g}), 453 \mathrm{~K}$. 
Even under these conditions, oligomeric cellulose and glucose were less stable than at lower temperatures, evidenced by the continual decrease in vibrational intensity at $\tilde{v}=1071$ and $1026 \mathrm{~cm}^{-1}$. A gradual increase in intensity of vibrations at $\tilde{v}=$ 1710 and $1586 \mathrm{~cm}^{-1}$ indicated the accumulation of small quantities LA and HMF. HPLC analysis of the solution and solids after the reaction revealed that approximately $36 \%$ of the original cellulose was converted with $25.7 \%$ selectivity to glucose. LA and HMF were formed with 7 and $5 \%$ selectivity, respectively. The remainder of the material consisted of humins and unconverted cellulose ( $55.6 \%$ selectivity) and small quantities of acetic acid, formic acid, fructose, mannose, and 2-furaldehyde ( $7 \%$ selectivity together).

\section{Conclusions}

Solid acid-catalyzed hydrolysis represents an attractive method for the valorization of cellulose into glucose and other valuable platforms chemical. In situ ATR-IR spectroscopy is a powerful technique that can be used to monitor the transformations that occur during this process, which includes the formation of the reaction products and by-products. The most relevant species, which include glucose and glucose oligomers, fructose, HMF, and several acids, are readily observed and generally distinguishable based on differences in vibrational characteristics even in the presence of water under elevated temperatures and pressures. In the case of regenerated cellulose with the activated carbon catalyst, relatively short reaction times $(\approx 5 \mathrm{~h})$ at $423 \mathrm{~K}$ minimized the formation of glucose degradation products.

A combination of analytical and spectroscopic results revealed key aspects of the catalytic system. The hydrolysis of cellulose in the absence of a catalyst is slow, but still observable by using ATR-IR spectroscopy. The presence of a solid-acid catalyst significantly increases the rate of cellulose hydrolysis to form glucose oligomers, the continued hydrolysis of which eventually results in the accumulation of monomeric glucose. The formation of other by-products proceeds through the isomerization of glucose to fructose, which is less hydrothermally stable and rapidly reacts to form by-products, such as HMF. The formation of HMF and other subsequent degradation products, which include LA and formic acid, are also significantly accelerated by the presence of solid-acid catalysts. Therefore, operation at reduced glucose residence times results in improved glucose selectivity. Harsher conditions (i.e., higher temperatures and the presence of stronger acid catalysts) tend to result in the increased formation of acid products and decreased glucose selectivity. Microcrystalline Avicel cellulose hydrolysis requires harsh conditions; therefore, higher acid content and lower glucose selectivity are typically observed during the hydrolysis of Avicel cellulose relative to regenerated cellulose, which is more susceptible to hydrolysis at lower reaction temperatures. Minimizing the contact to metal in the reactor, especially at higher temperatures, is important to decrease the formation of degradation products.

\section{Experimental Section}

The cellulose used was microcrystalline Avicel PH 101 (Fluka, particle size $\approx 50 \mu \mathrm{m}$ ), and the ionic liquid used was 1-butyl-2-methylimidazolium chloride (BMImCl, Basionic ST 70, purity $\geq 95 \%$ ). Both were purchased from Sigma-Aldrich. Sulfated zirconia (SZR) was supplied by Saint-Gobain NorPro, and activated carbon CAP SUPER was kindly provided by Norit. The carbon was activated using phosphoric acid with an acid density of approximately $1.1 \mathrm{mmol}$ $\mathrm{H}^{+}$equivalents per gram catalyst as determined by using titration. A suspension of $40 \mathrm{mg} \mathrm{mL}^{-1}$ per $\mathrm{g}$ of activated carbon in demineralized water resulted in a solution of $\mathrm{pH}$ 2.3. All compounds were used without further purification or pretreatment, unless stated otherwise.

For cellulose pretreatment, $\mathrm{BMImCl}$ was heated at $423 \mathrm{~K}$ for $2 \mathrm{~h}$ under a nitrogen flow before the addition of cellulose. After an incubation period of $30 \mathrm{~min}$, heating ceased, and the cellulose was precipitated by adding 5 parts $(\mathrm{v} / \mathrm{v})$ of hot demineralized water (>353 K). The liquid $\mathrm{BMImCl}$ solutions were stirred at all times. The solutions were stored at $4{ }^{\circ} \mathrm{C}$ overnight to allow the precipitate to settle. The precipitates were separated by centrifugation (Thermo Scientific SL 40R, $10 \mathrm{~min}$ at $4000 \mathrm{rpm}$ ) and washed three times with hot $(>353 \mathrm{~K})$ and once with cold demineralized water. The resultant solid is referred to as regenerated cellulose. The moisture content of the regenerated cellulose was adjusted to $95 \%$, which resulted in a colloidal suspension that was stable for weeks. The suspension was stored at $277 \mathrm{~K}$ until tested.

Hydrolysis reactions were conducted in a $40 \mathrm{~mL}$ Parr stainless steel autoclave equipped with an MT ReactIR $45 \mathrm{~m}$ attenuated total reflection (ATR)-IR sentinel with a diamond probe. To prevent catalytic breakdown of some of the products by the reactor wall at elevated temperatures $(>443 \mathrm{~K})$, a Teflon insert was required. The temperature was monitored by using a thermocouple, and stirring was conducted by using a magnetic driver equipped with an impellor at approximately $750 \mathrm{rpm}$. The head space of the reactor was flushed with nitrogen prior to heating $\left(2 \mathrm{Kmin}^{-1}\right)$. All experiments were performed at autogenous pressure. After the reaction, the solids were separated by centrifugation. The conversion of cellulose was calculated by the weight difference of cellulose before and after reaction. The supernatants were analyzed for typical products, such as glucose, fructose, HMF, furfural, formic acid, LA, and acetic acid (HPLC, Agilent 1100 series, equipped with an IR analyzer, UV detector and a Biorad AMINEX HPX-87H column). Compounds were identified by using elution times and quantified by using calibration standards. The solid residue was dried ( $323 \mathrm{~K}$, vacuum) until no further mass change was observed. The products yields are defined as the molar ratio of carbon in the product over the mol of carbon in the charged cellulose $(\times 100 \%)$.

\section{Acknowledgements}

J.Z. and B.M.W. gratefully thank the National Science Foundation International Research Fellowship Program for support of this research under Award No. 0856754. This research has been performed within the framework of the CatchBio program. The authors gratefully acknowledge the support of the Smart Mix Program of the Netherlands Ministry of Economic Affairs and the Netherlands Ministry of Education, Culture and Science. Thanks are also extended to Pieter C. A. Bruijnincx for many useful discussions. 
Keywords: cellulose - glucose - heterogeneous catalysis hydrolysis • ir spectroscopy

[1] R. D. Perlack, L. L. Wright, A. F. Turhollow, R. L. Graham, B. J. Stokes, D. C. Erbach, U. S. Department of Energy, Biomass as Feedstock for a Bioenergy and Bioproducts Industry: The Technical Feasibility of a Billion-Ton Annual Supply, 2005.

[2] Renewable Energy Road Map 2007, http://europa.eu/legislation_summaries/energy/renewable_energy/127065_en.htm.

[3] J. J. Bozell, J. E. Holladay, D. Johnson, J. F. White, Top Value Added Candidates from Biomass, Volume I: Results of Screening for Potential Candidates from Sugars and Synthesis Gas, Pacific Northwest National Laboratory: Richland, WA, 2007.

[4] J. J. Bozell, G. R. Petersen, Green Chem. 2010, 12, 539-554.

[5] Kirk-Othmer Encyclopedia of Chemical Technology, $4^{\text {th }}$ Ed. (Ed.: J. Kroschwitz), Wiley, New York, 1993, 5, 476-496.

[6] R. P. Swatloski, S. K. Spear, J. D. Holbrey, R. D. Rogers, J. Am. Chem. Soc. 2002, 124, 4974-4975.

[7] H. B. Zhao, J. E. Holladay, H. Brown, Z. C. Zhang, Science 2007, 316, $1597-1599$.

[8] F. Tao, H. Song, L. Chou, ChemSusChem 2010, 3, 1298-1303.

[9] F. Tao, H. Song, L. Chou, Carbohydr. Res. 2011, 346, 58-63.

[10] Y. Su, H. M. Brown, X. Huang, Z. C. Zhang, Appl. Catal. A: Gen. 2009, 361, $117-142$.

[11] M. B. Turner, S. K. Spear, J. D. Holbrey, R. D. Rogers, Biomacromolecules 2004, 5, 1379- 1384.

[12] H. Zhao, C. L. Jones, G. A. Baker, S. Xia, O. Olubajo, V. N. Person, J. Biotechnol. 2009, 139, 47-54.

[13] M. Kitano, D. Yamaguchi, S. Suganuma, K. Nakajima, H. Kato, S. Hayashi, M. Hara, Langmuir 2009, 25, 5068-5075.

[14] A. Onda, T. Ochi, K. Yanagisawa, Green Chem. 2008, 10, 1033-1037.

[15] R. Rinaldi, F. Schüth, ChemSusChem 2009, 2, 1096-1107.
[16] J. Pang, A. Wang, M. Zheng, T. Zhang, Chem. Commun. 2010, 46, 6935 6937.

[17] S.-J. Kim, A. A. Dwiatmoko, J. W. Choi, Y.-W. Suh, D. J. Suh, M. Oh, Biore sour. Technol. 2010, 101, 8273-8279.

[18] J. Hegner, K. C. Pereira, B. DeBoef, B. L. Lucht, Tetrahedron Lett. 2010, 51, $2356-2358$.

[19] A. Takagaki, C. Tagusagawa, K. Domen, Chem. Commun. 2008, $5363-$ 5365.

[20] S. Suganuma, K. Nakajima, M. Kitano, Y. Daizo, H. Kato, S. Hayashi, M. Hara, J. Am. Chem. Soc. 2008, 130, 12787-12793.

[21] B. Girisuta, L. P. B. M. Janssen, H. J. Heeres, Chem. Eng. Res. Des. 2006 $84,339-349$.

[22] J. B. Binder, A. V. Cefali, J. J. Blank, R. T. Raines, Energy Environ. Sci. 2010, 3, $765-771$

[23] K. Kervinen, M. Allmendinger, M. Leskelä, T. Repo, B. Rieger, Phys. Chem. Chem. Phys. 2003, 5, 4450-4454.

[24] K. Kervinen, H. Korpi, J. G. Mesu, F. Soulimani, T. Repo, B. Rieger, M. Leskelä, B. M. Weckhuysen, Eur. J. Inorg. Chem. 2005, 2591 -2599.

[25] J. Zakzeski, P. C. A. Bruijnincx, B. M. Weckhuysen, Green Chem. 2011, 13, $671-680$.

[26] Z. Zhang, G. E. Ewing, Anal. Chem. 2002, 74, 2578-2583.

[27] H. Shinzawa, S.-I. Morita, K. Awa, M. Okada, I. Noda, Y. Ozaki, H. Sato, Appl. Spectrosc. 2009, 63, 501-506.

[28] A. Watanabe, S. Morita, S. Kokot, M. Matsubara, K. Fukai, Y. Ozaki, J. Mol. Struct. 2006, 799, 102-110.

[29] G. Socrates, Infrared and Raman Characteristic Group Frequencies: Tables and Charts, Wiley, New York, 2001

[30] Q. Xiang, Y. Y. Lee, R. W. Torget, Appl. Biochem. Biotechnol. 2004, 115, $1127-1138$

Received: October 10, 2011

Published online on February 7, 2012 
